a high standard. Options for extending the service to nursing home settings are being explored.

\section{P124 IMPROVING END OF LIFE CARE FOR PATIENTS WITH DEMENTIA}

${ }^{1}$ Nicola Parkes, ${ }^{1}$ Gillian Cross, ${ }^{2}$ Andrea Whitfield. 'Trinity Hospice, Blackpool, UK," Blackpool Teaching Hospital, UK

10.1136/bmjspcare-2013-000591.146

Introduction Dementia is predominantly a disease of later life but there are at least 15000 people under 65 yrs who have the illness. Symptom relief is often missed as these patients are unable to communicate effectively.

Aims of the Project

- Improve the quality of End of life Care for people who are diagnosed with dementia admitted to hospital

- Increase the awareness of staff caring for these patients

- Promote advance care planning for people with dementia

- Support families of people suffering from dementia with decision making

Methods A flow chart was designed and coloured to reflect the local End of Life Care Model. There was close liaison with trust staff about developments in dementia care within the acute setting and teaching materials were developed to train staff on caring for patients with dementia in their last days of life.

Results The Trust collated results from the dementia screening tool used to asses every patient over $75 \mathrm{yrs}$; it demonstrated an Increase in knowledge for staff in trust to recognise dementia as a terminal disease.

Discussion The project aimed to support patients with dementia from diagnosis to bereavement. The work was a collaboration between the Acute Trust and local hospice services. Work streams were formed to develop care pathways, integrated working and shared learning. The trust adopted the butterfly scheme which the CNS team promoted when visiting patients with dementia and raise awareness of importance of advanced care planning for these patients.

Conclusion Improving the knowledge of staff around caring for patients that are dying who have dementia improves their ability to effectively observe and manage symptoms, improves the quality of care and job satisfaction whilst improving outcomes and relieving distress for patients and families.

\section{P125 PILOTING THE DESIGN AND DELIVERY OF A FIVE-DAY COURSE ON END OF LIFE CARE FOR PEOPLE WITH DEMENTIA. A JOINT VENTURE}

Laura Myers, Mary Mahoney. St Catherine's hospice, Crawley, UK

\subsection{6/bmjspcare-2013-000591.147}

The drivers for this project included National guidance and policy, such as the Prime Minister's Challenge (Department of Health (DH) 2012) and "Living Well with Dementia (DH, 2009) and the National end of life care strategy (DH, 2008), Regional influences were Dementia Local Implementation Groups (DLIG) and "Dementia-friendly communities". The development of the course was funded through the Regional Innovation Fund (RIF). The hospice is revising the organisational strategy, with a view to reaching more people who are dying and their families, through enhanced joint working.

The aim was to bring professionals together, sharing their specialist expertise to develop and deliver a five-day course to a wide variety of health and social care providers.

Initial work centred on accessing appropriate health and social care providers to help to design and deliver the course.. The group met regularly (over a period of six months), initially to establish the framework of the course and, subsequently, to design and agree the course content, intending to use facilitators from a range of disciplines.

A pilot five-day course was delivered in the Spring of 2013, attended by a diverse group of participants, including staff from mental health, learning disability, hospices, care homes, and community settings as well as a care commissioner. The course was facilitated by specialists from mental health, social care training, Alzheimer's society, specialist palliative care, care home setting, palliative medicine and hospice education, as well as a person with dementia. An assortment of teaching and learning techniques were used to encourage shared learning. Electronic evaluation has provided evidence of participants' learning.

This course has the potential to enable care providers to learn more about end of life care for people with dementia, reaching more people in all settings, in line with National, regional and organisational strategy.

\section{P126 RESPITE CARE REFRAMED - A PALLIATIVE CARE SERVICE FOR THE FUTURE}

Diane Laverty. St Joseph's Hospice, London, United Kingdom

10.1136/bmjspcare-2013-000591.148

Respite care is currently disregarded as an essential component of palliative care despite interest on the part of Government and others to support home based end of life care and to increase access for people with chronic degenerative conditions.

During the last 2 years an East London hospice has delivered an updated model of respite care that recognises and responds to new emerging groups of users and seeks to enable more people to remain at home during their illness.

A new, nurse led service, headed by a nurse consultant, has been established which delivers planned respite care. The service proactively seeks to support people with non malignant conditions including young adults undergoing transition from children to adult services.

This shift results in time being given to a multi professional review of the patient and carer's needs as a basis for establishing an updated care plan, designed to enrich the patient's life and maintain their function and wellbeing. Access to other hospice services is also made available. It is particularly effective when the patient is admitted in a relatively stable state and can consider / negotiate different approaches to care in a safe environment. Seven beds are now open to provide this care. Development of this new model has required a different culture of care, development of staff skills and new working relationships within and outwith the hospice.

The service has been well used (average occupancy 80\%). Review of activity data reveals a higher than average use of these beds by people with non cancer conditions (47\%) and increasing utilization of young people undergoing transition (12 episodes). User satisfaction is high and the impact of care, measured through SKIPP is positive. Carer feedback, via focus groups 